\title{
Sertraline with or without interpersonal psychotherapy reduces dysthymia symptoms over psychotherapy alone
}

\author{
Browne G, Steiner M, Roberts J et al. Sertaline and / or interpersonal psychotherapy for patients with dysthymic disorder in \\ primary care: 6-month comparison with longitudinal 2-year follow-up of effectiveness and costs. Journal of Affective \\ Disorders 2002 April; 68:317-30.
}

\section{QUESTION: For people with dysthymia, what are the long-term costs and effects of combining sertraline with interpersonal psychotherapy in primary care?}

\section{Design}

Randomised 6 month trial with 18 month follow up (allocation concealed). The outcome assessor was blind to group allocation.

\section{Setting}

Primary care university-affiliated Health Services Organisation in Ontario, Canada.

\section{Participants}

707 adults with DSM-IV dysthmic disorder. All were aged 18-74 years (mean 42 years; $68 \%$ female). People with a chronic or acute episode of a major depressive disorder were eligible. Exclusion criteria were previous sertraline use; hypersensitivity to selective serotonin reuptake inhibitors; acute suicide risk; depot neuroleptic drugs within 6 months; participation in other trials within 1 month; use of sertonergic drugs; primary or secondary diagnosis of a psychotic disorder; clinically significant or unstable medical conditions; pregnancy; lactation, and women of childbearing age not using reliable contraception or planning pregnancy within 25 months.

\section{Intervention}

Participants received 6 months of sertraline alone, interpersonal psychotherapy alone or sertraline plus psychotherapy. The initial dose of sertraline was $50 \mathrm{mg}$ per day, adjusted at 1-2 week intervals to a maximum of $200 \mathrm{mg}$ per day. The interpersonal psychotherapy group received up to 12 1-hour sessions (average number attended 10). After 6 months, participants chose to continue, discontinue or, in the case of the psychotherapy group, start sertraline treatment. The follow up rate was $83 \%$ at 6 months and $74 \%$ at 2 years.

\section{Main outcome measures}

Reduction in depressive symptoms using the Montgomery Asberg Depression Rating Scale (MADRS) at 6 months, 1 year and 2 years ( $\geq 40 \%$ reduction in MADRS scores); cost of treatment based on counselor time, medications, health and social services and indirect costs.

\section{Main results}

At 6 months there was a significant difference in mean MADRS scores and treatment response (table). Those receiving sertraline (with or without interpersonal psychotherapy) had reduced depressive symptoms compared to psychotherapy alone. These differences held after 2 years $(\mathrm{p}=0.03)$. There was no difference in the effect of sertraline alone versus combined with psychotherapy. Psychotherapy was associated with lower health and social service-use costs. Total per person costs at 2 years were $\$ 7866$ for sertraline alone, $\$ 7386$ for sertraline plus psychotherapy and $\$ 5657$ for psychotherapy alone (amounts in Canadian dollars, 2000).

\section{Conclusions}

After long-term follow up, sertraline (alone and in combination with interpersonal psychotherapy) is more effective than psychotherapy alone for reducing symptoms of dysthymia.

Sources of funding: Medical Research Council of Canada Pharmaceutical Manufacturers Association of Canada; Pfizer Canada Inc.

For correspondence: G Browne, Faculty of Health Sciences,

McMaster University, Canada.

Reduction in depressive symptoms after sertraline, interpersonal psychotherapy or combined treatment at 6 months

\begin{tabular}{lcccc} 
Outcome & $\begin{array}{c}\text { Sertraline } \\
(\mathbf{n}=196)\end{array}$ & $\begin{array}{c}\text { Sertraline plus } \\
\text { psychotherapy } \\
(\mathbf{n}=212)\end{array}$ & $\begin{array}{c}\text { Psychotherapy } \\
(\mathbf{n}=178)\end{array}$ & p-value \\
\hline Mean MADRS scores & 14.3 & 14.9 & 16.8 & $0.025^{\star}$ \\
\hline $\begin{array}{l}\text { Responding (40\% reduction } \\
\text { in MADRS score) }\end{array}$ & $60 \%$ & $58 \%$ & $47 \%$ & $0.021^{*}$ \\
\hline
\end{tabular}

* The psychotherapy group had more depressive symptoms and lower response to treatment compared with both the sertraline and combined groups.

\section{COMMENTARY}

Dysthymia is a persistent, long-standing condition so it is important to evaluate treatment effects over the longer term. In this study, participants could continue, stop or switch to their treatment(s) of choice during follow up. The findings are robust at 6 months, but the longer-term findings could be questioned because $26 \%$ had dropped out or were lost to follow up at 2 years. Additional treatments, including concomitant antidepressants, were not prohibited. Participants were also offered, and in most cases received, antidepressants for the full 2 years, whereas psychotherapy was only available for the first 6 months. It is perhaps unrealistic to expect 2 years of continuous psychotherapy, but the difference in treatment intensity should be noted.

How should we use these findings in clinical practice? The study suggests that antidepressants reduce dysthymic symptoms in the longer term, but that interpersonal psychotherapy reduces costs. There was no difference in the clinical efficacy of sertraline plus psychotherapy compared with sertraline alone, but overall costs were lower in the combined group due to reduced health and social service use. Combining pharmacotherapy and psychotherapy may therefore be the most clinically and resource-effective option.

One of the study's primary outcomes is cost effectiveness, yet there is no genuine control group against which to assess incremental treatment costs and benefits. The treatment groups have almost identical ratios of cost-to-effect, suggesting that the interventions offer similar value for money. However the study cannot tell us whether these treatments offer better value for money than usual care. Even with a usual care group, a larger sample would be needed to detect significant differences in cost-effectiveness. This is an ongoing problem in the economic evaluation of mental health interventions, not least as a result of variation in mean costs of care. Such variability is not reported in this study, perhaps because the authors' use a non-parametric test of difference. Non-parametric tests may be inappropriate for assessing cost differences since they do not compare arithmetic means. ${ }^{1}$

In summary, the authors suggest that clinicians should offer combined pharmacotherapy and psychotherapy for dysthymia in primary care. The clinical applicability of these findings is strengthened because of the 'real world' design, but assumptions about cost effectiveness may be flawed.

Dr Dan Chisholm Honorary Senior Lecturer Health Services Research Department, Institute of Psychiatry London, United Kingdom

1 Thompson S, Barber JA. How should cost data in pragmatic randomised trials be analysed? $B M J$ 2000; 320: 1197-200. 